\title{
FORMATION OF REVERSE MICELLES AND EXTRACTION OF ALUMINUM AND BERYLLIUM BY MIXTURE OF BIS(2-ETHYLHEXYL) PHOSPHORIC ACID AND DINONYLNAPHTHALENE SULFONIC ACID
}

\author{
MiKI MASHIMO, HiRoshi SATO AND \\ ISAO KOMASAWA \\ Department of Chemical Science and Engineering, Graduate School of \\ Engineering Science, Osaka University, Toyonaka 560
}

Key Words: Reverse Micelle, D2EHPA, DNNSA, Synergistic Effect, Extraction

\begin{abstract}
The formation of reverse micelles consisting of a mixture of bis(2-ethylhexyl) phosphoric acid (D2EHPA) and dinonylnaphthalene sulfonic acid (DNNSA) and its effect on the extraction of aluminum and beryllium have been studied. The total concentration of aggregates in the organic phase was measured by the vapor pressure osmometry (VPO) method. The average aggregation number for DNNSA micelle in $\boldsymbol{n}$-hexane was found to be 16. In the D2EHPA-DNNSA mixed system, the VPO result indicates the existence of dimeric D2EHPA and mixed reverse micelles consisting of both extractants. The mean number of D2EHPA molecules included in a mixed reverse micelle was determined by the relative concentrations of D2EHPA and DNNSA. The contributions of the reverse micelles and dimeric D2EHPA molecules to the extraction of metals were also evaluated. The distribution ratio obtained by the micelles increases substantially through inclusion of D2EHPA molecules into the micelles, with this inclusion causing a synergistic extraction of the metals in the mixed system.
\end{abstract}

\section{Introduction}

In the previous work (Mashimo et al., 1997), the extraction of aluminum and beryllium was studied using dinonylnaphthalene sulfonic acid (DNNSA), bis(2-ethylhexyl) phosphoric acid (D2EHPA) and their mixture as extractants. DNNSA in $n$-hexane was found to form reverse micelles with a critical micelle concentration (CMC) as low as $1 \times 10^{-5} \mathrm{M}$. In a mixture with D2EHPA, the CMC value for mixed reverse micelle formation was shifted to a higher value dependent on the concentration of D2EHPA. Here, free dimeric D2EHPA molecules (not involved in the micelles) and the mixed reverse micelles existed in organic solution. The mixed reverse micelles, however, showed a strong synergistic effect in the extraction of both metal ions, making the separation of both metals difficult.

There are several reports dealing with extraction systems involving reverse micelles. Harada et al., (1990) studied systems containing sodium bis(2ethylhexyl) sulfosuccinate (AOT) as surfactant and D2EHPA or 5,8-diethyl-7-hydroxy-6-dodecanone oxime (LIX63) as extractant, based on the spectroscopic difference existing between the organic copper complex and reverse micelles containing copper. In the AOT-D2EHPA system, the concentration of the copper complex and that of the

* Received on January 9, 1997. Correspondence concerning this article should be addressed to H. Sato. micelles containing copper in the mixed system were identical to the concentrations observed in the D2EHPA or AOT single systems, respectively. No synergistic extraction occurred and no mixed micelles were formed. In the AOT-LIX63 system, however, the synergistic effect was observed, but the contributions of the free extractant and micelles to the total extraction were not separated.

Knowledge of the structure and concentrations for the mixed micelles and free extractant is a first priority in order to understand the extraction mechanism for an extraction system involving reverse micelles. The total concentration of the aggregates can be estimated by vapor pressure osmometry (VPO), and there are some reports on VPO measurement for the D2EHPA-DNNSA system. However, the diluent or aqueous phase condition, in most cases, is not identical to that used in the metal extraction experiments. Based on concentrations calculated from VPO results, Van Dalen et al. (1978) estimated the contributions of DNNSA single micelles, D2EHPA-DNNSA mixed micelles, and free D2EHPA to the extraction of americium in the D2EHPA-DNNSA system. However, $n$-hexane was used as diluent for the VPO experiments whereas $n$ dodecane was employed for the extraction experiments. Other works show that the aggregation number of the reverse micelles formed with DNNSA depends both on the diluent and on the countercation of DNNSA (Markovits and Choppin, 1973; Chiarizia et al., 1976; Osseo-Asare and Keeney, 1980). 
The structure of reverse micelles in DNNSA single and D2EHPA-DNNSA mixed systems was investigated in the present study. The total concentration of the aggregates in solution was measured by the VPO technique. The sample was equilibrated with the same aqueous solution as used in the metal distribution experiments, and the concentrations of the free dimeric D2EHPA and the mixed micelles were estimated. From this, the individual contributions of the free D2EHPA and of the micelles to the overall extraction of both aluminum and beryllium are able to be calculated, and the extraction behavior of the mixed reverse micelles is discussed.

\section{Experimental}

The solutions and procedure for the measurement of extraction equilibria are the same as those described previously (Mashimo et al., 1997). The total concentration of the aggregates in the organic phase was measured by vapor pressure osmometry (VPO) using a Corona (Ibaraki, Japan) 114 molecular weight apparatus. Benzil supplied by Wako Pure Chemical Industries (Osaka, Japan) was used as a standard. An organic solution containing D2EHPA and DNNSA in $n$-hexane and an aqueous solution containing $0.005 \mathrm{M}$ metal ion, $0.17 \mathrm{M}$ sulfate ion and $0.093 \mathrm{M}$ sodium ion were shaken for $1-3$ hours at $298 \pm 0.5 \mathrm{~K}$ to attain extraction equilibrium for the metals and water. The equilibrium $\mathrm{pH}$ value was about 1. The solution was then centrifuged for 5 minutes at $2500 \mathrm{rpm}$ to obtain phase separation. The organic phase was then analyzed using the VPO apparatus at $310 \mathrm{~K}$.

\section{Results and Discussion}

\subsection{Concentration of aggregates in single and mixed systems}

The aggregation number and the concentration of the reverse micelles were calculated from the total concentration of the aggregates in the organic phase, as measured by the vapor pressure osmometry (VPO). Figure 1 shows the results obtained both for the DNNSA single system and the D2EHPA-DNNSA mixed system for a constant feed D2EHPA analytical (monomeric) concentration of $0.01 \mathrm{M}$. The left hand ordinate axis, $\Delta E$, represents the VPO voltage change which is proportional to the total concentration of aggregates in the solution, $C_{\mathrm{A}}$. The proportionality factor is determined from the relationship between $\Delta E$ and the concentration of standard material (benzil) as shown by the square points. The value was $63000 \mu \mathrm{V} / \mathrm{M}$ in this case, and the $C_{\mathrm{A}}$ scale calculated from this value is shown via the right hand axis.

The results for the DNNSA single system are plotted by means of circles. With a critical micelle

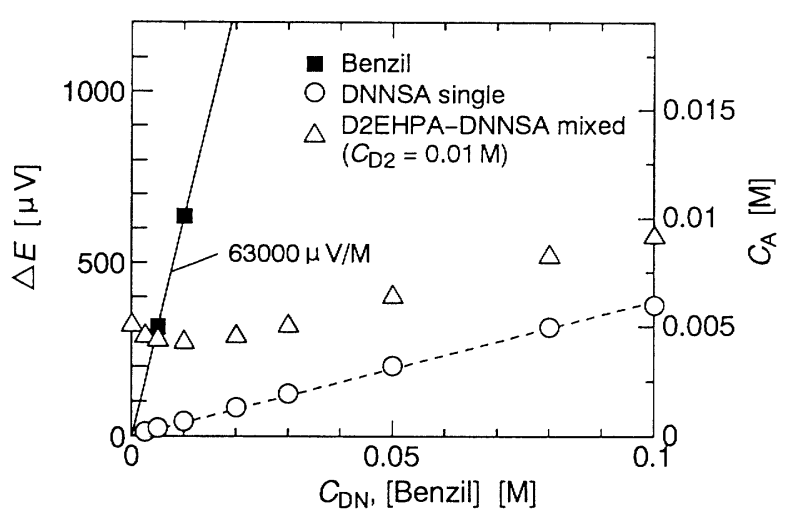

Fig. 1 Effect of DNNSA analytical concentration $\left(C_{\mathrm{DN}}\right)$ on results of VPO measurement for DNNSA single and D2EHPA-DNNSA mixed systems at constant feed D2EHPA analytical concentration of $0.01 \mathrm{M}$; $0.005 \mathrm{M} \mathrm{Al}, 310 \mathrm{~K}$

concentration (CMC) of DNNSA estimated as $1 \times 10^{-5} \mathrm{M}$ (Mashimo et al., 1997), reverse micelles are thus formed for all measurements. The data points lie on a straight line through the origin, indicating that the aggregation number of the DNNSA micelle is independent of DNNSA concentration, with the aggregation number calculated to be $16.3 \pm 0.8$ from the slope of the line. This value is almost identical to the value of 17 reported for $n$-hexane solution (OsseoAsare and Keeney, 1980).

However, a linear relationship between the total aggregate concentration, $C_{\mathrm{A}}$, and the DNNSA analytical concentration, $C_{\mathrm{DN}}$, in the mixed system in the presence of constant D2EHPA analytical concentration of $0.01 \mathrm{M}$ is not obtained. The datum point on the left hand axis $\left(C_{\mathrm{DN}}=0 \mathrm{M}\right)$ shows an aggregate concentration in the $0.01 \mathrm{M} \mathrm{D} 2 \mathrm{EHPA}$ single system. The value obtained was $0.0049 \mathrm{M}$, indicating that D2EHPA exists as a dimer in the organic phase as previously reported (Komasawa et al., 1981). Increasing the DNNSA concentration in the range 0 $0.01 \mathrm{M}$ results in the total aggregate concentration falling below $0.0049 \mathrm{M}$. This indicates that the mixed aggregate containing three or more D2EHPA molecules and some DNNSA molecules is formed in this region. In the presence of $0.01 \mathrm{M} \mathrm{D} 2 \mathrm{EHPA}$, the value of CMC of DNNSA has been reported to be $4 \times 10^{-4} \mathrm{M}$ (Mashimo et al., 1997). Thus the observed decrease in the total aggregate concentration is caused by the formation of mixed micelles containing three or more D2EHPA molecules. When however, the DNNSA analytical concentration increases above the value of $0.01 \mathrm{M}$, the total aggregate concentration increases continuously. This is caused by an increase in the concentration of the micelles due to the increase in the DNNSA concentration.

Figure 2 shows the effect of the D2EHPA 


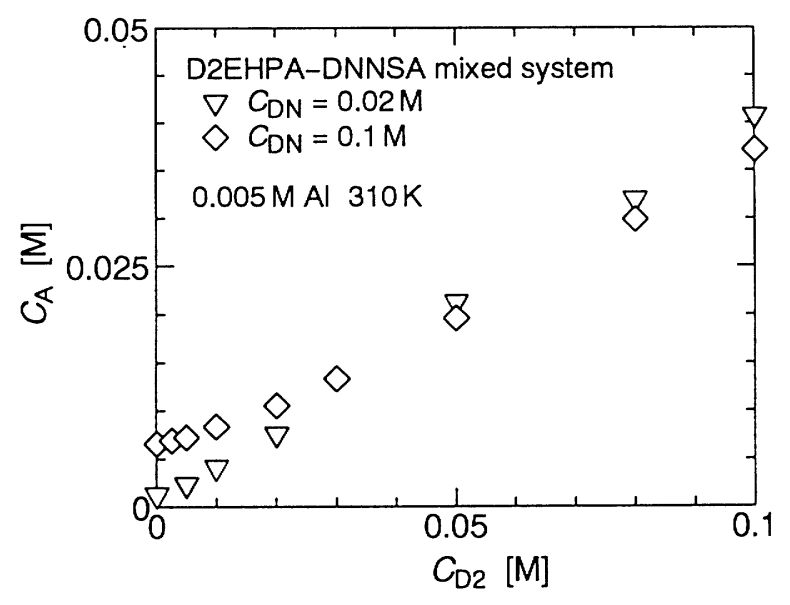

Fig. 2 Effect of D2EHPA analytical concentration $\left(C_{\mathrm{D} 2}\right)$ on total aggregate concentration $\left(C_{\mathrm{A}}\right)$ for D2EHPADNNSA mixed system; $0.005 \mathrm{M} \mathrm{Al}, 310 \mathrm{~K}$

analytical concentration on total aggregate concentration for the mixed system. The aggregate concentration increases continuously with increasing D2EHPA concentration, with a slope of less than 0.5, thus indicating that the number of D2EHPA molecules in a mixed micelle increases with increasing D2EHPA concentration. The composition of the mixed micelles therefore changes with varying concentrations of DNNSA and D2EHPA.

\subsection{Concentration and composition of mixed micelles}

The present VPO study was used to estimate quantitatively the concentration and composition of the micelles. In a previous study (Mashimo et al., 1997), it was found that there was free dimeric D2EHPA (not involved in the micelles) as well as the reverse micelles containing various numbers of D2EHPA and DNNSA molecules. The total concentration of aggregates $\left(C_{\mathrm{A}}\right)$ is expressed by the sum of the concentration of the free dimeric D2EHPA $\left(C_{2 \mathrm{D} 2}\right)$ and that of the reverse micelles $\left(C_{\mathrm{mic}}\right)$.

$$
C_{\mathrm{A}}=C_{2 \mathrm{D} 2}+C_{\mathrm{mic}}
$$

The contribution of water dissolved in the continuous phase on the VPO signal was neglected since there was no significant difference in the signals for water extracted $n$-hexane and pure $n$-hexane. Assuming that on average a micelle contains $m$ DNNSA molecules and $n$ D2EHPA molecules, the analytical concentrations of D2EHPA $\left(C_{\mathrm{D} 2}\right)$ and DNNSA $\left(C_{\mathrm{DN}}\right)$ molecules in the solution are expressed as

$$
\begin{aligned}
& C_{\mathrm{D} 2}=n C_{\mathrm{mic}}+2 C_{2 \mathrm{D} 2} \\
& C_{\mathrm{DN}}=m C_{\mathrm{mic}}
\end{aligned}
$$
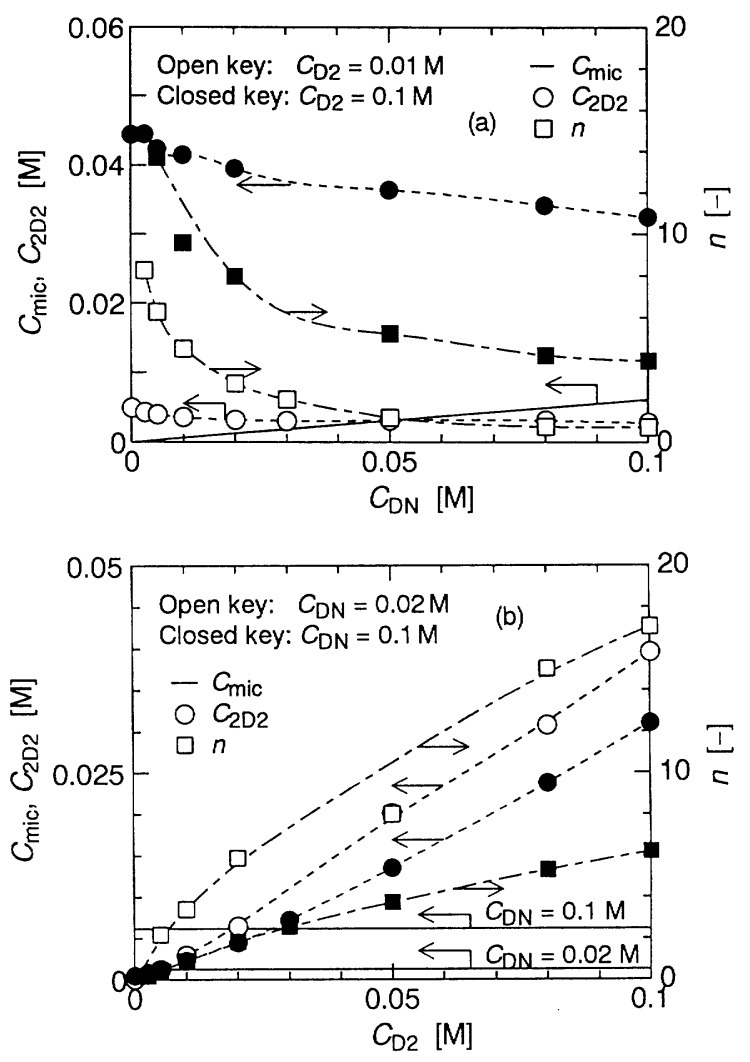

Fig. 3 Concentration of micelles $\left(C_{\mathrm{mic}}\right)$, concentration of free dimeric D2EHPA $\left(C_{2 \mathrm{D} 2}\right)$ and average number of D2EHPA molecules in a micelle $(n) ; 0.005 \mathrm{M} \mathrm{Al}$; (a) effect of DNNSA analytical concentration at constant D2EHPA analytical concentration; (b) effect of D2EHPA analytical concentration at constant DNNSA analytical concentration

Thus,

$$
\begin{aligned}
& C_{\text {mic }}=C_{\mathrm{DN}} / m \\
& C_{2 \mathrm{D} 2}=C_{\mathrm{A}}-C_{\mathrm{DN}} / m \\
& n=\left(m / C_{\mathrm{DN}}\right)\left(C_{\mathrm{D} 2}-2 C_{\mathrm{A}}\right)+2
\end{aligned}
$$

From Eq. (6), the number of D2EHPA molecules $(n)$ in a micelle is greater than two for $C_{\mathrm{A}}$ less than $C_{\mathrm{D} 2} / 2$. This is observed in the case of $C_{\mathrm{D} 2}=0.01 \mathrm{M}$ and $0 \mathrm{M}<C_{\mathrm{DN}}<0.03 \mathrm{M}$ as shown in Fig. 1 .

Assuming that $m$ is independent of the number of D2EHPA molecules in the micelle, the value of $m$ may be estimated as 16 from the VPO result for the DNNSA single system. This assumption has been made in the previous analysis performed by Van Dalen et al. (1974). The values of $C_{\text {mic }}, C_{2 \mathrm{D} 2}$ and $n$ thus calculated are presented in Fig. 3, showing that the concentration of the micelles is controlled by the DNNSA analytical concentration. An increase in the feed D2EHPA concentration brings about an increase in the number of D2EHPA molecules 
composing the micelle as well as the concentration of free dimeric D2EHPA. The maximum value of $n$ observed under the present conditions is 17 , and as many D2EHPA molecules as DNNSA molecules can be included in a micelle in certain cases.

\subsection{Extraction of metals by dimeric D2EHPA and by the micelles}

Both the free dimeric D2EHPA and the mixed micelles consisting of D2EHPA and DNNSA can extract metals. The contributions of the two species to the extraction were estimated both from the present VPO study and the previous extraction equilibrium study (Mashimo et al., 1997). The total concentration of metal ions in the organic phase $\left(\overline{C_{\mathrm{M}}}\right)$ is the sum of the concentration of metal ions extracted by the free D2EHPA $\left(\overline{C_{\mathrm{M}, \mathrm{D} 2}}\right)$ and that by the mixed micelles ( $\overline{C_{\mathrm{M}, \mathrm{mic}}}$.

$$
\overline{C_{\mathrm{M}}}=\overline{C_{\mathrm{M}, \mathrm{D} 2}}+\overline{C_{\mathrm{M}, \mathrm{mic}}}=D C_{\mathrm{M}}
$$

where $D$ is the observed distribution ratio. The extraction equilibria for the free D2EHPA and the micelles are expressed as

$$
\begin{aligned}
& \overline{C_{\mathrm{M}, \mathrm{D} 2}}=D_{\mathrm{D} 2} C_{\mathrm{M}} \\
& \overline{C_{\mathrm{M}, \mathrm{mic}}}=D_{\text {mic }} C_{\mathrm{M}} \\
& D=D_{\mathrm{D} 2}+D_{\text {mic }}
\end{aligned}
$$

where $D_{\mathrm{D} 2}$ and $D_{\text {mic }}$ are the distribution ratios for the free D2EHPA and for the micelles, respectively.

The extraction equilibrium formulations for aluminum and beryllium with D2EHPA (HR) were established as (Mashimo et al., 1997)

$$
\begin{array}{r}
\mathrm{Al}^{3+}+2 \overline{(\mathrm{HR})_{2}} \rightleftarrows \overline{\mathrm{AlR}_{3} \mathrm{HR}}+3 \mathrm{H}^{+} \\
K_{\mathrm{ex}}=0.147 \mathrm{M} \\
\mathrm{Be}^{2+}+2 \overline{(\mathrm{HR})_{2}} \rightleftarrows \overline{\mathrm{BeR}_{2}(\mathrm{HR})_{2}}+2 \mathrm{H}^{+} \\
K_{\mathrm{ex}}=49.5
\end{array}
$$

Assuming that the extraction equilibrium with free D2EHPA is not influenced by the presence of the micelles, the contribution of free D2EHPA to the metal extraction $\left(D_{\mathrm{D} 2}\right)$ can be calculated by Eqs. (11) and (12) and the free D2EHPA concentration in the mixed extractant system.

The observed distribution ratios obtained with a single extractant, D2EHPA or DNNSA, and their mixture are cited from the previous work. These are shown by the lines in Fig. 4(a) -(d). The contribution of the free D2EHPA as estimated in the present VPO study is also plotted. This is seen to decrease with an increase in the concentration of DNNSA. The degree of the decrease is notable for the case of $0.01 \mathrm{M}$ feed concentration of D2EHPA, since in this case, the degree of the reduction of the free D2EHPA molecules due to the formation of the mixed micelles is greater as shown in Fig. 3(a). The other contribution of the mixed micelles is calculated as the difference between the total distribution ratio, $D$, and the contribution of the free D2EHPA, $D_{\mathrm{D} 2}$, and this is also plotted in Fig. 4(a)-(d). This contribution is seen to increase with increasing DNNSA concentration and becomes dominant in the total distribution ratio. The two contributions are comparable only in the case of beryllium extraction with $0.1 \mathrm{M}$ feed concentration of D2EHPA. This is as expected, since free D2EHPA is a very good extractant for beryllium and $65 \%$ of the feed D2EHPA still exists as free dimers in the presence of 0.1 M DNNSA, as shown in Fig. 3(a).

The synergistic effect of the mixed reverse micelles is seen most clearly in the case of aluminum extraction with $0.1 \mathrm{M}$ D2EHPA. With a DNNSA concentration greater than $2.5 \times 10^{-3} \mathrm{M}$, the observed distribution ratio in the mixed system is much greater than those for the D2EHPA and DNNSA single systems, as shown in Fig. 4(a). The synergistic effect is actually brought about by the inclusion of D2EHPA molecules into the DNNSA micelles to form the mixed micelles. This effect is expressed by the ratio of the distribution ratio of the mixed micelles to the ratio of the single DNNSA micelles, $D_{\text {mic }} / D_{\mathrm{DN}}$. The ratio is plotted in Fig. 5 as a function of the number of D2EHPA molecules included in a micelle $(n)$. The ratios for both aluminum and beryllium are seen to increase rapidly with increasing $n$ value. The D2EHPA molecules included in the micelles have a much stronger complexing power to aluminum ions as well as beryllium ions than the free dimeric D2EHPA molecules. This approximately equal and very powerful complexing power for both metal ions makes the separation of the two metals non-feasible.

Single DNNSA reverse micelles have been found to extract metal ions by a cation exchange mechanism (Mashimo et al., 1997). The extraction mechanism with the mixed reverse micelles can also be considered. As shown in Fig. 4(a), for aluminum extraction with $0.1 \mathrm{M}$ feed concentration of D2EHPA and $0.01 \mathrm{M}$ DNNSA, the contribution of the mixed micelles is 27 times greater than the contribution of the free D2EHPA and thus the mechanism is controlled by the mixed micelles. The slope analysis applied to the system is shown in Fig. 5 of the previous study. The distribution ratio was inversely proportional to the third power of the hydrogen ion concentration in the absence of sodium ions and thus the extraction mechanism was expressed by a cation exchange mechanism, as with the single DNNSA micelles. 

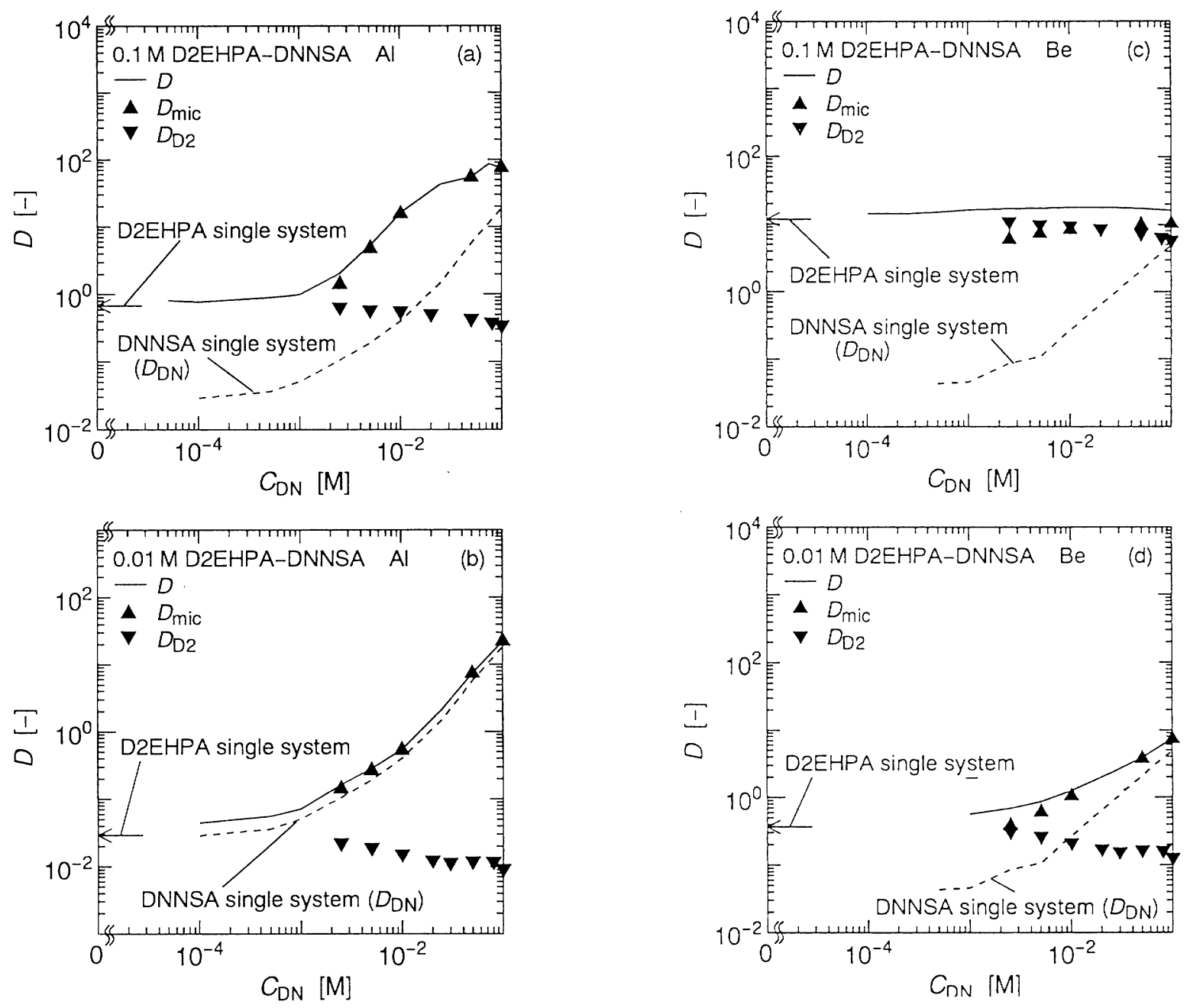

Fig. 4 Distribution ratios obtained with D2EHPA or DNNSA single systems and mixture of both extractants cited from Mashimo et al., 1997 and contributions of free D2EHPA and micelles to distribution ratio; (a) $0.005 \mathrm{M} \mathrm{Al}, C_{\mathrm{D} 2}=0.1 \mathrm{M}$; (b) $0.005 \mathrm{M} \mathrm{Al}, C_{\mathrm{D} 2}=0.01 \mathrm{M}$; (c) $0.005 \mathrm{M} \mathrm{Be}, C_{\mathrm{D} 2}=0.1 \mathrm{M}$; (d) $0.005 \mathrm{M} \mathrm{Be}$, $C_{\mathrm{D} 2}=0.01 \mathrm{M}$

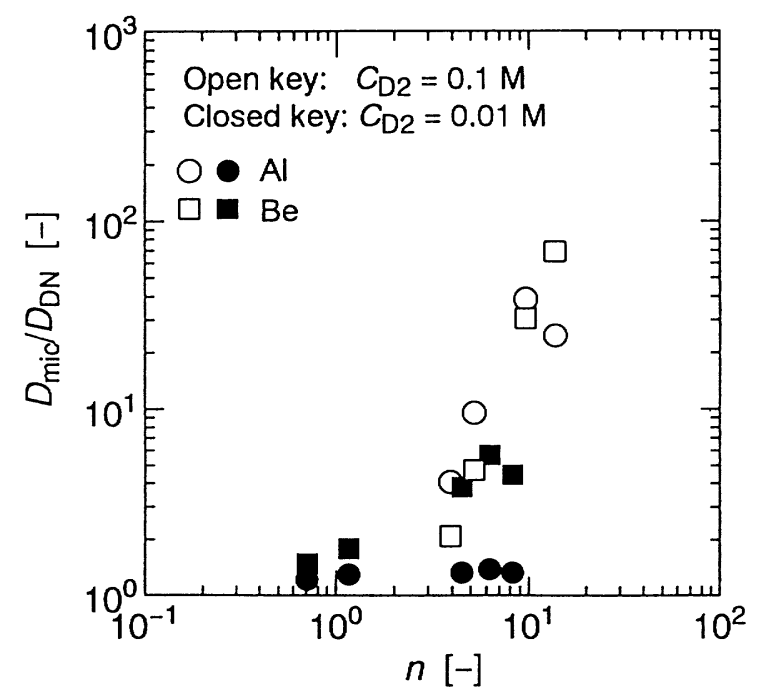

Fig. 5 Effect of number of D2EHPA molecules included in a mixed micelle on enhancing complexing power

\section{Conclusion}

The formation of reverse micelles in a mixture of bis(2-ethylhexyl) phosphoric acid (D2EHPA) and dinonylnaphthalene sulfonic acid (DNNSA) and the effect of micellar formation on extraction of aluminum and beryllium are studied.

The aggregation number of the micelle in $n$ hexane was measured by vapor pressure osmometry method. The average aggregation number of a DNNSA single micelle was found to be 16 . In the D2EHPA-DNNSA mixed system, mixed micelles consisting of both extractants and dimeric D2EHPA exist. The mean number of D2EHPA molecules included in a micelle was calculated by assuming that the aggregation number of DNNSA in the mixed micelle is the same as that in the DNNSA single micelle and that the free D2EHPA exists as a dimer. 
The number increases both with increasing D2EHPA concentration and decreasing DNNSA concentration, and the maximum value observed in the present study is nearly the same as that for DNNSA.

The contributions of the D2EHPA-DNNSA mixed micelles and the free dimeric D2EHPA in the extraction of metals were evaluated. The extraction mechanism for the mixed micelles is cation exchange, as is the case for DNNSA single micelles. The complexing power of the mixed micelles is very, and almost equally, strong for both aluminum and beryllium, and this makes the separation of the two metals difficult.

\section{Acknowledgment}

The authors wish to express their thanks to Profs. J. Shibata and S. Nishimura, Department of Chemical Engineering, Kansai University for use of the vapor pressure osmometry apparatus. M.M. gratefully acknowledges financial support from the Morishita Zintan Scholarship Foundation.

\begin{tabular}{|c|c|c|c|}
\hline \multicolumn{4}{|c|}{ Nomenclature } \\
\hline$C_{2 \mathrm{D} 2}$ & $=$ & molar concentration of dimeric D2EHPA & [M] \\
\hline$C_{\mathrm{A}}$ & $=$ & total concentration of aggregates in the solutio & \\
\hline$C_{\mathrm{D} 2}$ & $=$ & analytical (monomer) concentration of D2EH & PA \\
\hline & & molecules in the solution & [M] \\
\hline$C_{\mathrm{DN}}$ & $=$ & $\begin{array}{l}\text { analytical (monomer) concentration of DNNS } \\
\text { molecules in the solution }\end{array}$ & A \\
\hline$C_{\mathrm{M}}$ & $=$ & molar concentration of metal ion & [M] \\
\hline$\overline{C_{\mathrm{M}, \mathrm{D} 2}}$ & $=$ & $\begin{array}{l}\text { molar concentration of metal ion extracted by } \\
\text { free D2EHPA }\end{array}$ & [M] \\
\hline$\overline{C_{\mathrm{M}, \text { mic }}}$ & $=$ & $\begin{array}{l}\text { molar concentration of metal ion extracted } \\
\text { by reverse micelles }\end{array}$ & [M] \\
\hline$C_{\text {mic }}$ & $=$ & molar concentration of reverse micelles & {$[\mathrm{M}]$} \\
\hline$D$ & $=$ & observed distribution ratio & {$[-]$} \\
\hline$D_{\text {mic }}$ & $=$ & $\begin{array}{l}\text { contribution of reverse micelles to distribution } \\
\text { ratio }\end{array}$ & {$[-]$} \\
\hline$D_{\mathrm{D} 2}$ & $=$ & $\begin{array}{l}\text { contribution of free D2EHPA to distribution } \\
\text { ratio }\end{array}$ & \\
\hline
\end{tabular}

\begin{tabular}{|c|c|c|}
\hline$D_{\text {DN }}$ & $\begin{array}{l}=\text { distribution ratio observed in DNNSA single } \\
\text { system }\end{array}$ & {$[-]$} \\
\hline$m$ & $\begin{array}{l}=\text { average number of DNNSA molecules } \\
\text { composing a reverse micelle }\end{array}$ & {$[-]$} \\
\hline$n$ & $\begin{aligned}= & \text { average number of D2EHPA molecules } \\
& \text { composing a mixed micelle }\end{aligned}$ & {$[-]$} \\
\hline$\Delta E$ & $=\mathrm{VPO}$ voltage change & [V] \\
\hline $\begin{array}{l}{[\mathrm{l}]} \\
\text { overbar }\end{array}$ & $\begin{array}{l}=\text { concentration of species in the brackets } \\
=\text { species in organic phase }\end{array}$ & {$[\mathrm{M}]$} \\
\hline
\end{tabular}

\section{Literature Cited}

Chiarizia, R., P.R. Danesi, G.D'alessandro and B. Scuppa; "Interfacial Behavior of Dinonylnaphthalenesulfonic Acid at the Toluene- $\mathrm{HClO}_{4}$ Interface," J. Inorg. Nucl. Chem., 38, 1367-1369 (1976)

Harada, M., N. Shinbara, M. Adachi and Y. Miyake; "Liquid Membrane Operation with Aid of Microemulsion Separation of Metal Ions-," J. Chem. Eng. Japan, 23, 50-57 (1990)

Komasawa, I., T. Otake and Y. Higaki; "Equilibrium Studies of the Extraction of Divalent Metals from Nitrate Media with Di-(2ethylhexyl) Phosphoric Acid," J. Inorg. Nucl. Chem., 43, 33513356 (1981)

Markovits, G.Y. and G.R. Choppin; Ion Exchange and Solvent Extraction in a Series of Advances vol. 3, J. Marinsky and Y. Marcus ed., p. 51-81, Marcel Dekker, New York, U.S.A. (1973)

Mashimo, M., H. Sato, M. Ueda and I. Komasawa; "Extraction Equilibria of Aluminum and Beryllium from Sulfate Media by Mixture of Bis(2-ethylhexyl) Phosphoric Acid and Dinonylnaphthalene Sulfonic Acid," J. Chem. Eng. Japan, 30, 706-711 (1997)

Osseo-Asare, K. and M.E. Keeney; "Aspects of the Interfacial Chemistry of Nickel Extraction with LIX63-HDNNS Mixtures," Metallurgical Trans. B, 11, 63-67 (1980)

Van Dalen, A., K.W. Gerrisma and J. Wijkstra; "Inclusion of Some Organic Acid in Micelle of Dinonylnaphthalene Sulfonic Acid," J. Colloid Interface Sci., 47, 127-133 (1974)

Van Dalen, A., J. Wijkstra and K.W. Gerrisma; "Liquid-liquid Extraction of Complexed Metal Ions by Inclusion in Dinonylnaphthalene Sulfonic Acid Micelles," J. Inorg. Nucl. Chem., 47, 875-881 (1978) 